\title{
Sequencing of Tri- and Tetraantennary N-Glycans Containing Sialic Acid by Negative Mode ESI QTOF Tandem MS
}

\author{
Dijana Sagi and Jasna Peter-Katalinic \\ Institute for Medical Physics and Biophysics, University of Muenster, Muenster, Germany \\ Harald S. Conradt \\ Protein Glycosylation Research, German Research Center for Biotechnology, Braunschweig, Germany \\ Manfred Nimtz \\ Protein Structure Research, German Research Center for Biotechnology, Braunschweig, Germany
}

\begin{abstract}
Application of the negative mode electrospray ionization-quadrupole time-of-flight mass spectrometry (ESI QTOF) tandem MS for determination of substitution patterns by sialic acid and/or fucose and extention by additional LacNAc disaccharide units in single branches of multiantennary N-glycans from biological samples is described. Fragmentation patterns which can be obtained by low energy collision-induced dissociation (CID) using the QTOF instrument include cleavage ions, diagnostic for determination of antennarity and for specific structural features of single antennae. Systematic fragmentation studies in the negative ion mode were focussed toward formation of the D diagnostic ion relevant for assignment of 3and 6-antennae in complex N-glycans carrying three and four antennae in combination with epitope-relevant B- and C-type ions. For validation of this approach ESI QTOF fragmentation of the permethylated analogues was carried out in the positive ion mode. Using this strategy, products of in vitro glycosylation reactions were investigated in order to clarify some general aspects of $\mathrm{N}$-glycan acceptor specificity during biosynthesis. $\alpha 1-3$ fucosylation using GDPfucose along with a soluble form of the recombinant human $\alpha 1-3$ fucosyltransferase VI was carried out on tri- and tetraantennary precursors to test structural requirements for formation of $\mathrm{Le}^{\mathrm{x}}$ versus sLe ${ }^{\mathrm{x}}$ motifs. (J Am Soc Mass Spectrom 2002, 13, 1138-1148) (c 2002 American Society for Mass Spectrometry
\end{abstract}

A number of biologically relevant glycoproteins contain large N-linked complex carbohydrate moieties, proposed to be involved in specific interactions with other molecules and/or cell surfaces. Highly regulated biosynthetic pathways in which $\mathrm{N}$ acetylglucosaminyltransferases are incorporating GlcNAc residues on the mannotriose core are determining factors for the formation of a number of antennae in complex-type N-glycans [1]. In multiantennary glycans different antennae can show different extent of elongation by $\mathrm{N}$-acetyllactosaminyl repeats and number and site of modifications by sialic acid and fucose moieties, contributing to the microheterogeneity on the single

Published online August 19, 2002

Address reprint requests to Dr. J. Peter-Katalinic, Biomedical Analysis Group, Institute for Medical Physics and Biophysics, University of Muenster, Robert-Koch Str. 31, D-48149 Muenster, Germany. E-mail: jkp@unimuenster.de

This article was presented as an oral contribution at the 48th ASMS Conference on Mass Spectrometry and Allied Topics, Long Beach, California, USA, June 11-15, 2000. glycosylation site. Besides, the expression of a certain oligosaccharide epitope may be restricted to just one of the antennae present. In order to carry on a complete characterization of complex type $\mathrm{N}$-glycan mixtures obtained from glycoproteins after release by $\mathrm{N}$ glycanase, combined chromatographic, mass spectrometric and nuclear magnetic resonance spectroscopic approach can be mandatory, as demonstrated in the case of human erythropoietin (hEPO), a glycoprotein containing bi-, tri- and tetraantennary $\mathrm{N}$-glycan structures [2]. Its biological activity in terms of the clearance rate was positively correlated with the ratio of tetra- to biantennary N-glycans, where the hEPO with wellbranched tetraantennary glycans remained at higher levels in the plasma [3]. Particular attention is to be paid to identification of glycosylation patterns in terms of antennarity in glycoproteins from different organs, since they may express identical protein cores, carrying glycoforms of different antennarity and therefore different antennae structure and modifications by sialic acid and fucose in different type of linkages. It has been 
shown recently that CD52, a GPI anchored glycopeptide with a single N-glycosylation site, contains bi-, tri-, and tetraantennary glycoforms if isolated from human semen, where this from lymphocytes contains only tetraantennary ones $[4,5]$.

Fragmentation of carbohydrates by tandem mass spectrometric (MS/MS) methods in combination with fast atom bombardment (FAB) [6-8], electrospray (ESI) [9-13], or matrix-assisted laser desorption/ionization (MALDI) [14-16] has been extensively studied. Carbohydrates have been commonly submitted to chemical derivatization prior to MS analysis to increase the relative stability of sialic acid moiety during the desorption, or to increase the ionization degree. Permethylation has been the most frequently used derivatization followed by fragmentation in the positive ion mode [13, 17]. Underivatized carbohydrates carrying sialic acids were observed to give more intense mass spectra in the negative than in the positive ion mode. However, significantly less information on the fragmentation of negative ions from complex $\mathrm{N}$-glycans, particularly those carrying more then two antennae, is available than on the positive ones [18].

In our contribution an application of the negative mode ESI QTOF tandem MS is described for structure determination of branched N-glycans from biological samples. Fragmentation patterns obtained by low energy CID using the QTOF instrument include cleavage ions, diagnostic for determination of antennarity and for epitope formation on single branches. For validation of this approach ESI QTOF fragmentation of the permethylated analogues was carried out in the positive ion mode. Fragmentation patterns obtained indicate the validity of the strategy described here, particularly important for such cases, where only a limited amount of biological material, in the low pmole range, is available.

\section{Experimental}

\section{Preparation of N-Glycans}

$\alpha 2$-3sialylated oligosaccharides were isolated from recombinant human erythropoietin which was expressed in BHK-21 cells [19]. After detachment of the total $\mathrm{N}$-glycans with polypeptide N-glycanase, the oligosaccharide mixture was subjected to ion exchange chromatography on Mono Q [2] and the individual charged oligosaccharide fractions were pooled separately (asialo-, monosialo to pentasialo fraction). The trisialylated fraction was further resolved by $\mathrm{HPLC}$ on $\mathrm{NH}_{2}-$ bonded phase. Individual peaks were pooled and characterized by MALDI/TOF MS after desialylation and by methylation analysis.

\section{In vitro $\alpha 1-3 f u c o s y l a t i o n$ of the Trisialylated N-Glycans}

In vitro $\alpha 1-3$ fucosylation was performed as described previously [20] using GDP-fucose along with a soluble form of recombinant human $\alpha 1-3$ fucosyltransferase VI which has been shown to add peripheral fucose to asialo as well as to $\alpha 2-3$ sialylated N-glycans thus forming both the $\operatorname{Le}^{\mathrm{x}}$ as well the sLe ${ }^{\mathrm{x}}$ motifs [21].

Partially $\alpha 1-3$ fucosylated trisialylated tetraantennary oligosaccharides were passed over a $\mathrm{RP}-\mathrm{C}_{18}$-cartridge. High-pH anion-exchange chromatography with pulsed amperometric detection (HPAEC-PAD) analysis indicated a mixture of $\mathrm{N}$-glycans containing $0-3$ peripheral fucose residues as deduced from the 4 min decrease in $R_{t}$ upon introduction of each fucose residue.

Native $\mathrm{N}$-glycan samples were additionally desalted on the graphitized carbon column [22] prior to ESI MS. Mini cartridges were prepared by dismantling a normal-sized GlycoClean H cartridge (Oxford GlycoSciences, UK) and packing the material into disposable pipette tips as generally described earlier [23]. Prior to use, the columns were washed with three changes of 50 $\mu \mathrm{l} 80 \%$ acetonitrile (vol/vol) in $0.1 \%$ (vol/vol) TFA followed by $3 \times 100 \mu \mathrm{l}$ of water. The samples were dissolved in 30-50 $\mu \mathrm{l}$ of water and applied to the column. Salts were then washed off with $3 \times 100 \mu$ l of water. Glycans were eluted by the application of $3 \times 50$ $\mu \mathrm{l} 50 \%$ acetonitrile (vol/vol) in $0.1 \%$ (vol/vol) TFA. These fractions were collected and dried in vacuo. Permethylation of $\mathrm{N}$-glycans was performed as described previously [24].

\section{Nanoelectrospray Ionization-Quadrupole Time-of- Flight Mass Spectrometry}

N-glycans were analyzed by nanoESI MS and MS/MS using a quadrupole time-of-flight (Q TOF) mass spectrometer (Micromass, Manchester, UK) equipped with a nanoESI source as described previously [25]. Sialylated native $\mathrm{N}$-glycans were analyzed in the negative ion mode and permethylated in the positive. The capillaries for nanospray were produced in-house on a capillary puller (David Kopf Instruments, Tujunga, CA).The Nglycans were dissolved in methanol-water (1:1/vol:vol). The capillary voltage was approximately $1100 \mathrm{~V}$ and the cone voltage adjusted to generate appropriate ions (see Results).

\section{Results and Discussion}

\section{Considerations of Experimental Conditions for Analysis of Complex-Type N-Glycans}

A formation of molecular ions of different charge states and their abundance is directly dependent on the cone voltage in the ion source, where the intensity of ions at a lower charge state is decreased at lower cone voltages. On the other hand, at higher cone voltages a certain degree of in-source degradation takes place, giving rise to in-source decay (ISD) fragment ions. This holds particularly for less stable modifications on complextype N-glycans, N-acetylneuraminic acid (NeuAc), or fucose (Fuc) moieties. In consideration of this aspect it 
would be more appropriate to use lower cone voltage for sugar mapping in mixtures, since overlapping $\mathrm{m} / \mathrm{z}$ values of a molecular ion and an ISD fragment ion in spectra could eventually lead to incorrect structural interpretations in MS1 and in MS/MS mode.

Derivatization of native glycans by permethylation can contribute to increased stability of the substrate in the ion source, facilitating the choice of desorption conditions. In addition, the fragmentation patterns for single and for multiple bond cleavages can be distinguished. However, this option is to be considered only if sufficient material for analysis, e.g., $10-15$ pmoles, is available, and if the analysis time scale does allow it.

A further aspect to consider is the energy requirement for the fragmentation process under low energy CID conditions, which is a function of the charge state of the precursor ion. Although fragmentation patterns obtained from precursor ions of different charge states are generally similar, those of lower charge states require significantly higher collision energy for fragmentation. However, MS/MS data originating from precursor ions of higher charge states could result in rather complex ion patterns, sometimes difficult to assign unambiguously, because of potential overlapping fragment ions derived from different charge states in the observed $\mathrm{m} / \mathrm{z}$ area.

Considering these aspects specific for structural analysis of naturally occurring complex-type $\mathrm{N}$-glycans as native substrates a cone voltage of $50-70 \mathrm{~V}$ has been chosen as an optimum for mapping and sequencing in this study. Under these conditions molecular ions of triand tetraantennary $\mathrm{N}$-glycans were most abundant at higher charge states, e.g., 3-, and the formation of ISD fragment ions is compromised. For reduced and permethylated glycan samples cone voltages at $60-80 \mathrm{~V}$ were chosen.

\section{Fragmentation Patterns of Sialylated and Fucosylated Complex-Type N-Glycans}

Particular attention of this study was focussed to the sialylation and fucosylation patterns, in order to develop an approach for analysis of modifications on single branches of multiantennary substrates. A loss of sialic acid appears as the most frequent fragmentation reaction under the low energy CID conditions, so that the sialylated $\mathrm{B}$ and $\mathrm{C}$ ions characterizing the terminal epitopes are frequently of low abundance. Fucose moiety is considered to be less labile. It can be found as a core and peripheral substituent, respectively. Its presence on an antenna as a part of the terminal epitope can be determined from the tri- and tetrasaccharide B-ions. On the other hand, cross-ring fragment ions ${ }^{0,2} \mathrm{~A}$ and ${ }^{2,4} \mathrm{~A}$, resulting from the reducing termini of N-glycans, provide direct evidence for identification of the coreFuc in the chitobiose moiety. The $\mathrm{m} / \mathrm{z}$ difference between ${ }^{0,2} \mathrm{~A}_{7}$ and ${ }^{2,4} \mathrm{~A}_{7}$ ions of the N-glycan with the core-Fuc is $206 \mathrm{u}$, and that without is $60 \mathrm{u}$.

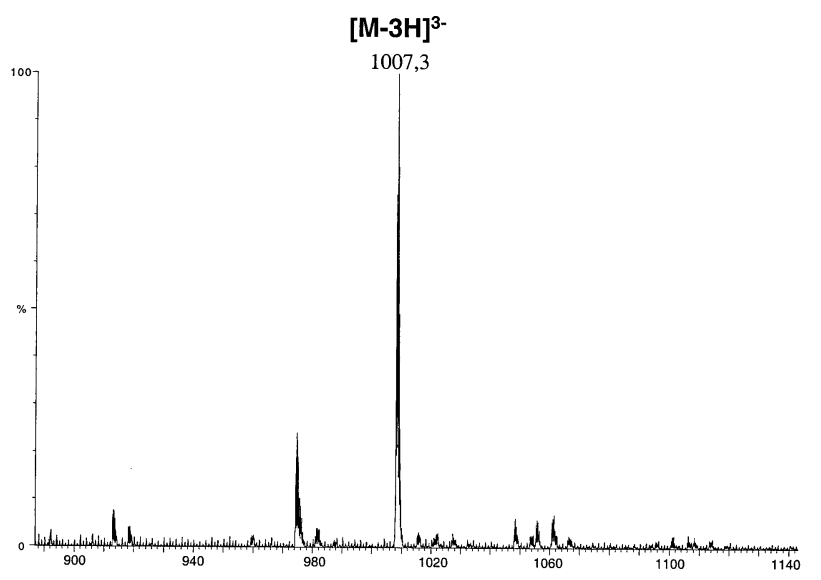

(a)

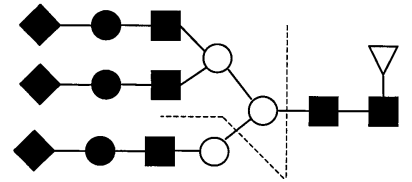

$\mathrm{C}_{4 \alpha} \quad 835,28$

$\mathrm{B}_{5} / \mathrm{Y}_{3 \alpha}($,D“ ion) $\quad 1636,56$

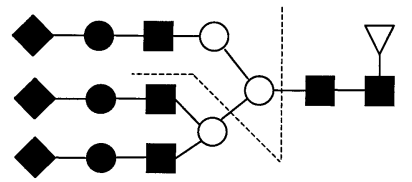

$\mathrm{C}_{4 \alpha} 1491,51$

$\mathrm{B}_{5} / \mathrm{Y}_{3 \alpha}($ (D“ ion) 980,33

(b)

Figure 1. Negative ion MS of a native triantennary N-glycan with a core fucose (a) and structures of its two possible isobaric glycoforms (b). Key to symbols: Filled square $=$ GlcNAc, filled circle $=$ galactose, open circle $=$ mannose, filled diamond $=$ sialic acid, open triangle $=$ fucose.

\section{Triantennary N-Glycan}

Triantennary N-glycans can exist in two isobaric forms, containing either a branched 3-linked or a branched 6-linked antenna. In Figure 1a the negative mode ESI MS of the N-glycans sample obtained from recombinant human erythropoietin [19] is depicted. Besides a few structurally uncorrelated ions of lower abundance, the major component is a triply charged ionic species assigned to a triantennary in the chitobiose $\alpha 1,6$-linked monofucosylated N-glycan. Two triantennary isomers containing either the branched 6-linked (Figure 1b, upper trace) or the branched 3-linked antenna (Figure $1 \mathrm{~b}$, lower trace) could be distinguished by their respective typical fragmentation patterns, in particular by their $\mathrm{C}_{4}$ and " $\mathrm{D}$ " fragment ions. $\mathrm{B}_{5} / \mathrm{Y}_{3 \alpha}$ or " $\mathrm{D}$ " ion (Figure $1 \mathrm{~b}$ ) is defined as a fragment ion formed by the loss of the 3-antenna together with the N-glycan chitobiose core. It has been postulated earlier as a reliable ion for distinction of the 3- and 6-antennae of N-linked glycans in the positive ion mode [26]. The difference in the relative stability of substituents attached to the 3-position versus the 6-position was observed previously by several authors [27-30]. 


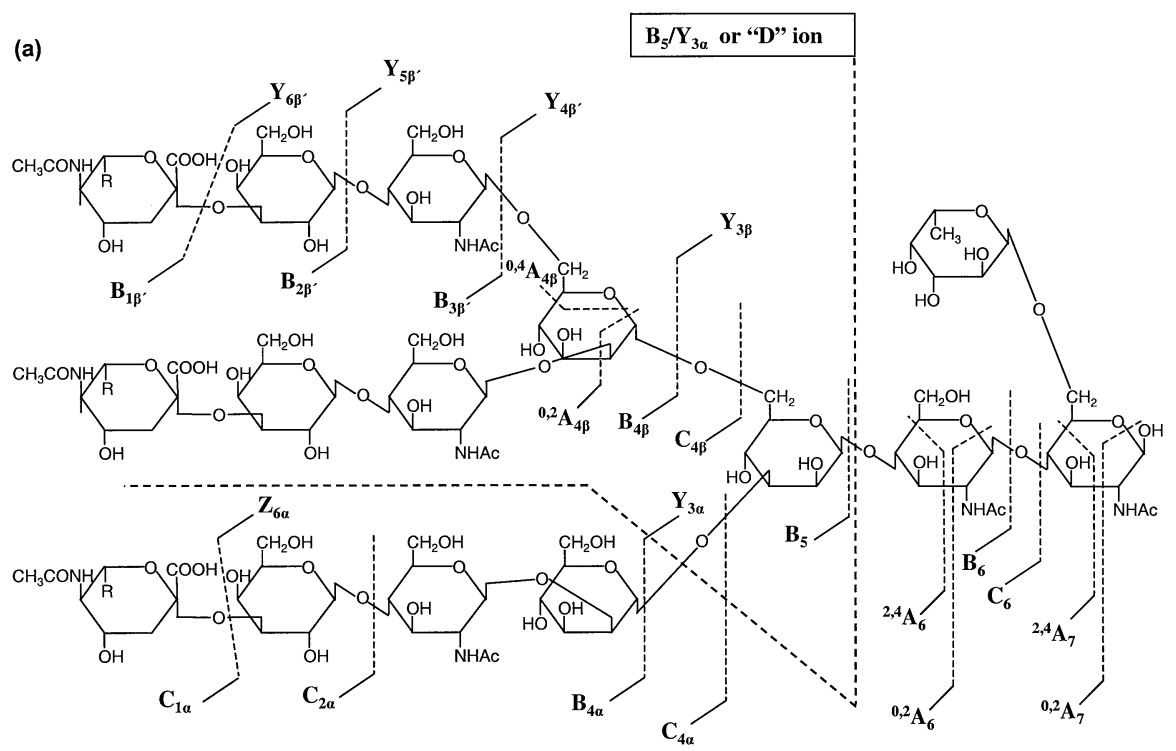

$\mathrm{R}=\mathrm{CHOH} \mathrm{CHOH} \mathrm{CH}_{2} \mathrm{OH}$

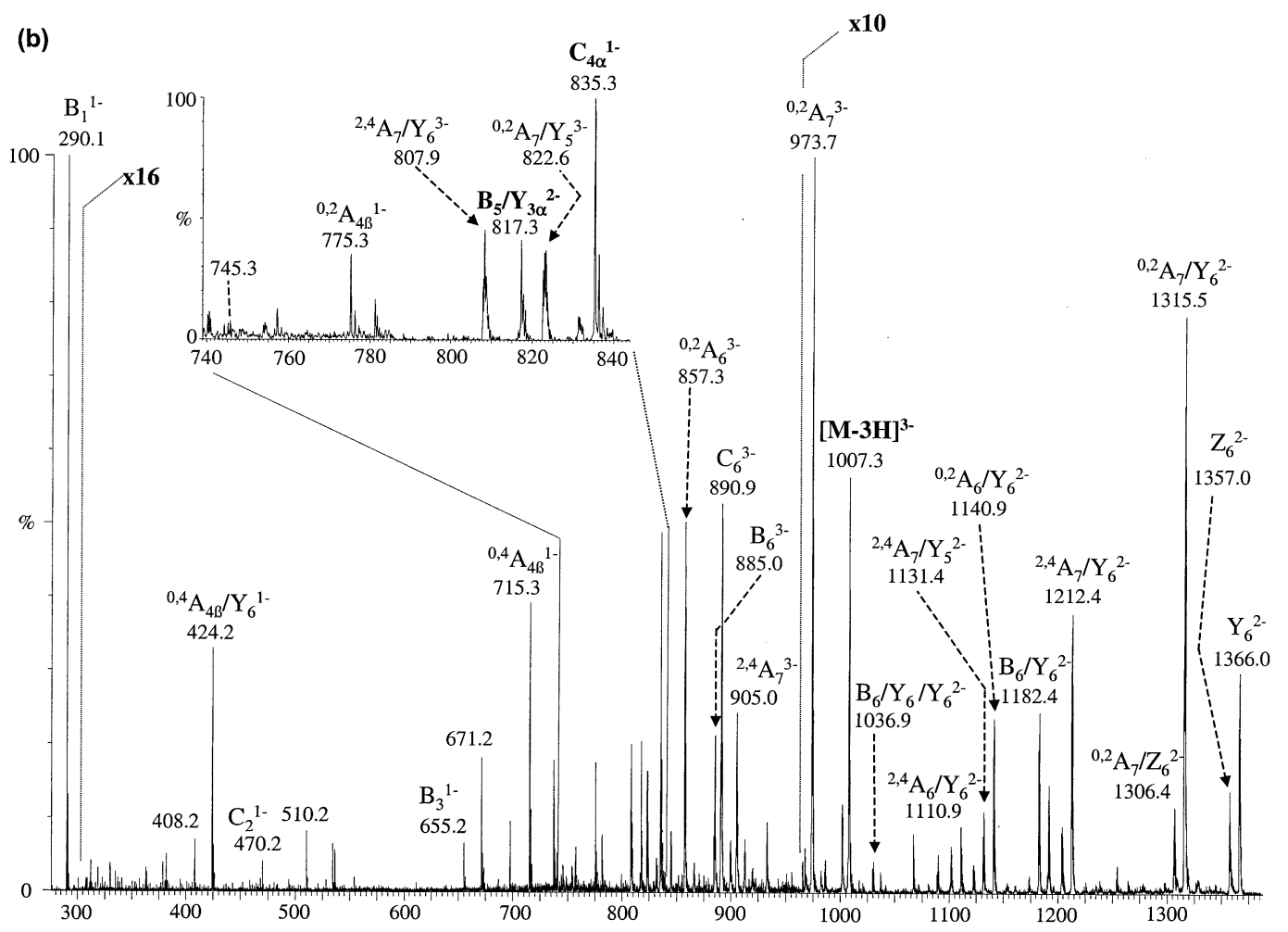

Figure 2. Triantennary complex type core-fucosylated N-glycan with branched 6-linked antenna: (a) Scheme of the fragment ion formation. (b) Negative ion MS/MS of its triply charged $[\mathrm{M}-3 \mathrm{H}]^{3-}$ molecular ion at $\mathrm{m} / \mathrm{z}$ 1007.3.

For fragmentation analysis in the negative ion mode MS/MS the $[\mathrm{M}-3 \mathrm{H}]^{3-}$ ion at $\mathrm{m} / z 1007.3$ was used as a precursor (Figure 2). Only the mass range up to $\mathrm{m} / \mathrm{z}$ $1400 \mathrm{u}$ showing higher charged state fragment ions is depicted, while the singly charged counterpart at higher $\mathrm{m} / \mathrm{z}$ values was much less abundant. The diagnostic ion for the assignment of the antennae, D-ion, appeared as a doubly charged species at $\mathrm{m} / \mathrm{z}$ 817.3.
According to this fragment ion the structure can be assigned to the triantennary $\mathrm{N}$-glycan containing a branched 6-linked antenna fucosylated in the chitobiose core (Figure $2 b$ ). Significantly high abundant $\mathrm{C}_{4 \alpha}$ fragment ion was at $m / z 835.3$ while the $C_{4 \beta}$ appeared as a doubly charged species at $m / z 745.3$. The $B_{5} / Y_{3 \beta}$ ion, which would represent the loss of the 6-antenna together with the chitobiose core, was not detected. Major 


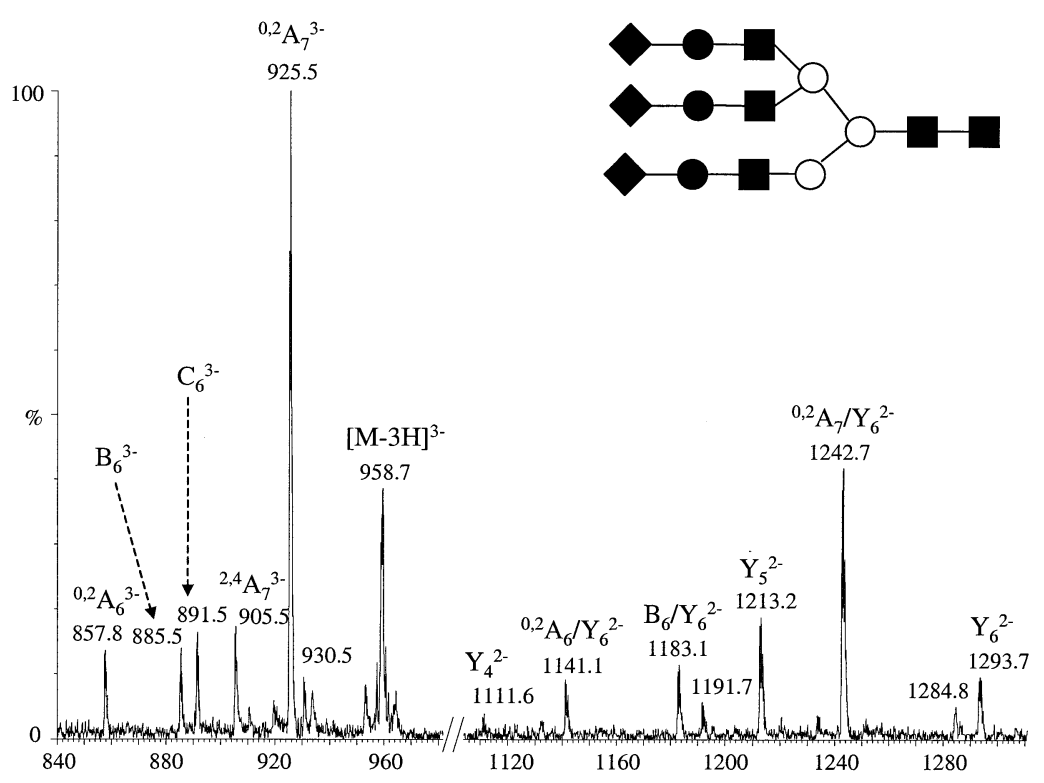

Figure 3. Negative ion MS/MS and the structure of the triantennary non core-fucosylated N-glycan. The mass ranges 840-980 and 1105-1310 are displayed. The precursor ion was the triply charged $[\mathrm{M}-3 \mathrm{H}]^{3-}$ ion at $\mathrm{m} / \mathrm{z} 958.7$.

sites of glycosidic cleavages were those between the GlcNAc residues of the chitobiose core, $\mathrm{C}_{6}$ and $\mathrm{B}_{6}$, and those at the sialic acid attachment sites, $\mathrm{Y}_{6}$ and $\mathrm{Z}_{6}$. Besides, fragment ions arising from cross-ring cleavages, ${ }^{0,2} \mathrm{~A}_{7},{ }^{2,4} \mathrm{~A}_{7}$, and ${ }^{0,2} \mathrm{~A}_{6}$, were observed. Internal fragments, formed by two or more cleavages of glycosidic bonds, like $\mathrm{B}_{6} / \mathrm{Y}_{6}$ and $\mathrm{B}_{6} / \mathrm{Y}_{6} / \mathrm{Y}_{6}$, or by a combination of glycosidic and cross-ring cleavages, like ${ }^{0,2} \mathrm{~A}_{7} /$ $\mathrm{Y}_{6}{ }^{0,2} \mathrm{~A}_{7} / \mathrm{Y}_{5},{ }^{2,4} \mathrm{~A}_{7} / \mathrm{Y}_{6},{ }^{2,4} \mathrm{~A}_{7} / \mathrm{Y}_{5}$, and ${ }^{0,2} \mathrm{~A}_{6} / \mathrm{Y}_{6}$, were also rather high abundant. All these ions were supportive for structural assignment in context of the 6-branched antennae.

The structure of the trisialo triantennary N-glycan analogue without core fucose has been investigated by fragmentation analysis using the molecular $[\mathrm{M}-3 \mathrm{H}]^{3-}$ ion at $m / z 958.7$ as a precursor (Figure 3). The absence of the core fucose was documented by the typical mass difference of $60 \mathrm{u}$ between the two triply charged ring cleavage ions ${ }^{0,2} \mathrm{~A}_{7}$ at $m / z 925.5$ and ${ }^{2,4} \mathrm{~A}_{7}$ at $m / z$ 905.5. In case of the fucosylated glycan, the mass difference between the same type of fragment ions, ${ }^{0,2} \mathrm{~A}_{7}$ at $\mathrm{m} / \mathrm{z}$ 973.7 and ${ }^{2,4} \mathrm{~A}_{7}$ at $m / z$ 905.0, was $206 \mathrm{u}$ (Figure 2). The mass difference related to this structural motif is useful for easy and fast diagnosis of the core fucose. Ions for assignment of antennae were not detected in this spectrum.

\section{Tetraantennary N-Glycan Containing Three Sialic Acids and One Peripheral Fucose}

Tetraantennary N-glycan containing three sialic acid moieties and an $\alpha 1,6$-linked core fucose was used as a substrate for in vitro fucosylation assay using fucosyltransferase VI (FT VI). FT VI has been previously reported to attach peripheral fucose moieties on sialy- lated as well as nonsialylated antenna of biantennary N-glycans with similar efficiency [20]. Preferred attachment sites in the multiantennary substrates used in this study, which offer both potential acceptor epitopes, were however, unknown. Following the enzymatic reaction a mixture of $\mathrm{N}$-glycans was analyzed by negative ion MS and a number of products, differing in degree of peripheral fucosylation, detected as triply and doubly charged molecular ions without and with one sodium exchanged (Figure 4).

In order to examine the efficiency of FT VI to fucosylate the sialylated and the nonsialylated antennae it was necessary to determine attachment sites of the peripheral fucose moieties added. To address this question fragment ions diagnostic for assignment of antennae and for distinction of isobaric structures according to their fragmentation patterns should serve as a tool for exploring the FT VI recognition specificity. The presence of the fucose substituent on a single antenna can be documented by $B_{3}$ or $C_{3}$ ions. Additionally, four possible $\mathrm{C}_{4 \alpha}$ or $\mathrm{B}_{4 \alpha}$ ions can provide further information for the Fuc and/or NeuAc branch location.

The triply charged molecular $[\mathrm{M}-3 \mathrm{H}]^{3-}$ ion at $\mathrm{m} / \mathrm{z}$ 1177.7, assigned to the $\mathrm{N}$-glycan with one peripheral Fuc, was selected for fragmentation analysis by MS/MS (Figure 5). Four $B_{3}$ ions, marked as $B_{3+F}, B_{3}, B_{3+F-N A}$, and $\mathrm{B}_{3-\mathrm{NA}}$, were observed as singly charged ions at $\mathrm{m} / \mathrm{z}$ 801.3, 655.2, 510.2, and 364.1, respectively. Diagnostic were the singly charged fragment ions, $C_{4 \alpha 2}$ at $\mathrm{m} / \mathrm{z}$ 1346.5 and $\mathrm{C}_{4 \alpha 3}$ at $\mathrm{m} / z$ 1200.4, beside those doubly charged, $C_{4 \alpha 1}$ at $m / z 754.3$ and $C_{4 \alpha 4}$ at $m / z$ 818.3. From the $m / z$ values of these ions it was concluded that on all four antennae substoichiometric amounts of fucose moieties were present. These results are in agreement with the previous reports on other substrates, suggest- 

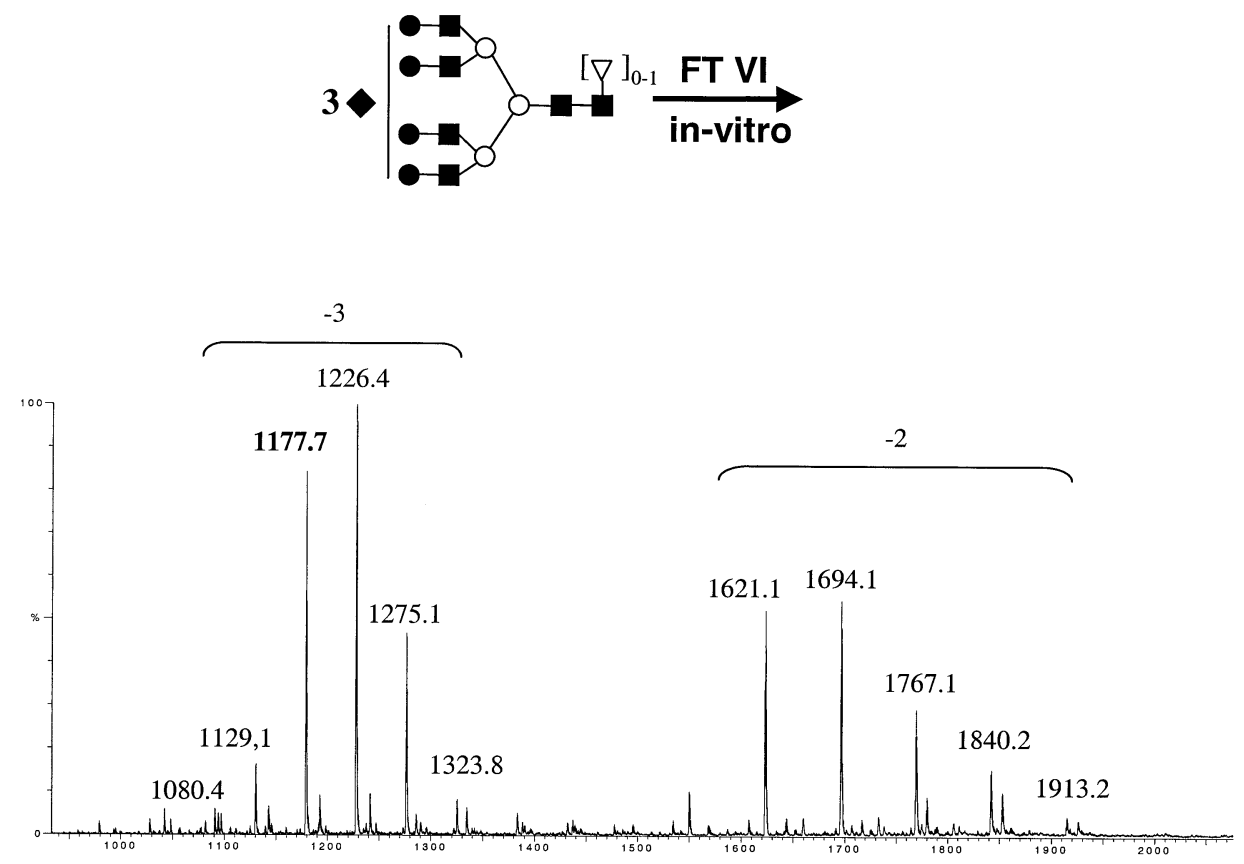

Figure 4. Negative ion MS of a native glycan mixture obtained after in vitro fucosylation with the fucosyltransferase VI (FT VI). The substrate for FT VI was a tetraantennary N-glycan containing three sialic acid moieties and the $\alpha 1-6$-linked core fucose.

ing that sialylated as well as nonsialylated antennae could be fucosylated by FT VI with similar efficiency [19].

Ambiguities related to a prompt loss of sialic acid residues attached to single antenna sites can lead to problems in distinguishing terminal fragment ions from internal ones. This holds for a singly charged ion at $\mathrm{m} / \mathrm{z}$ 364.1 which can be assigned either to the terminal fragment ion $\mathrm{B}_{3-\mathrm{NA}}$, or to the internal fragment ion $\mathrm{B}_{3} / \mathrm{Y}_{6}$. In order to provide additional structural evidence on sialylation the sample was derivatized by permethylation prior to the tandem MS analysis. If in the fragmentation analysis ions originating from the double cleavages occur, they can be assigned as such because of the relevant mass difference of $14 \mathrm{u}$ in their diagnostic ions, witnessing detachment of the NeuAc moiety during the ionization process.

Following these considerations, the same tetraantennary N-glycan sample was permethylated and submitted to fragmentation MS/MS analysis in the positive ion low-energy CID (Figure 6) using the triply charged molecular $[\mathrm{M}+3 \mathrm{Na}]^{3+}$ ion at $m / z 1482.1$ as a precursor. An almost complete set of $Y$-ions with sodium attached, except for $Y_{3}$ ions, was observed. Internal fragments formed by the loss of sialic acid moieties from Y-ions were observed at rather high abundance in the permethylated sample as well. Among B-ions, high abundant $\mathbf{B}_{1}$ oxonium fragment ion at $m / z 376.2$ was accompanied by sodiated fragment ion, at $m / z$ 398.2. The high abundance of nonsodiated B-ions could be explained by different cationization patterns of the chosen precursor molecular ion being enclosed with other potential precursor ions, unintentionally selected along for CID, e.g.,
$[\mathrm{M}+\mathrm{K}+\mathrm{Na}+\mathrm{H}]^{3+}$ at $m / z$ 1480.1. Higher mass B ions appeared only in form of their sodium adducts. Of particular interest were the $\mathrm{B}_{3}$ ions: All four of them, $\mathrm{B}_{3+\mathrm{F}}$ at $m / z 1021.4, \mathrm{~B}_{3}$ at $m / z 847.4, \mathrm{~B}_{3+\mathrm{F}-\mathrm{NA}}$ at $m / z$ 660.3, and $\mathrm{B}_{3-\mathrm{NA}}$ at $m / z 486.2$, were present in the spectrum as singly charged ions at relative high abundance (Figure 6). In addition, internal fragment ions $B_{3+F} / Y_{6}$ and $\mathrm{B}_{3} / \mathrm{Y}_{6}$ were easily identified in the permethylated substrate due to the mass difference of $14 \mathrm{u}$ in comparison with those arising from cleavage of a single glycosidic linkage, like $\mathrm{B}_{3+\mathrm{F}-\mathrm{NA}}$ and $\mathrm{B}_{3-\mathrm{NA}}$.

Using this double protocol, the final evidence about the NeuAc moiety attachment site was deduced from fragmentation patterns of both native and permethylated N-glycans, in particular from the appearance of the double cleavage ions.

Data collected in these experiments further allow a conclusion that there is no structural or conformational preference of single branches playing a role in fucosylation by FT VI.

\section{Tetraantennary N-Glycan with One Additional LacNAc Repeat Unit}

To explore the acceptor specificity for fucose attachment in relation with the chain length a tetraantennary $\mathrm{N}$-glycan containing one branch extended by an additional LacNAc repeat unit and one proximal fucose was also submitted to an in vitro fucosylation with the recombinant FT VI. On the branch with the LacNAc repeat unit extention, there are two potential sites for fucose attachment at both GlcNAc moieties. The prod- 
(a)
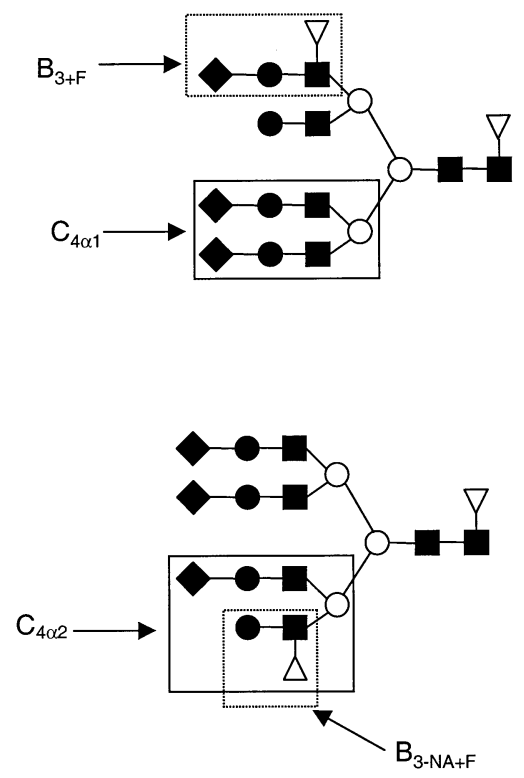

(b)

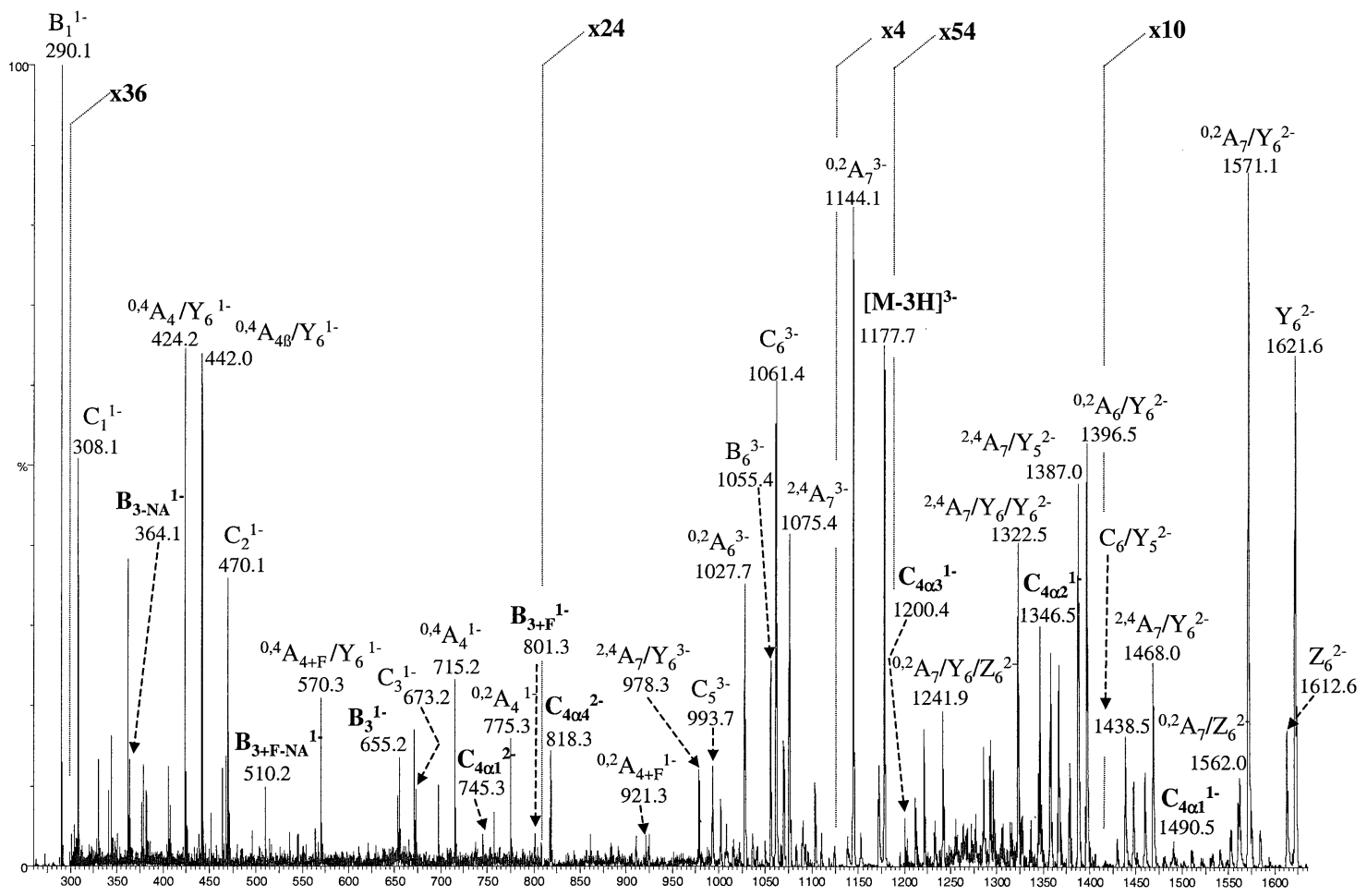

Figure 5. (a) Structures of diagnostic fragment ions for determination of peripheral fucose attachment in isobaric tetraantennary N-glycan structures carrying three sialic acid, one core and one peripheral fucose moieties. (b) Negative ion MS/MS of the same tetraantennary N-glycan. The precursor ion was the triply charged molecular $[\mathrm{M}-3 \mathrm{H}]^{3-}$ ion at $m / z$ 1177.7.
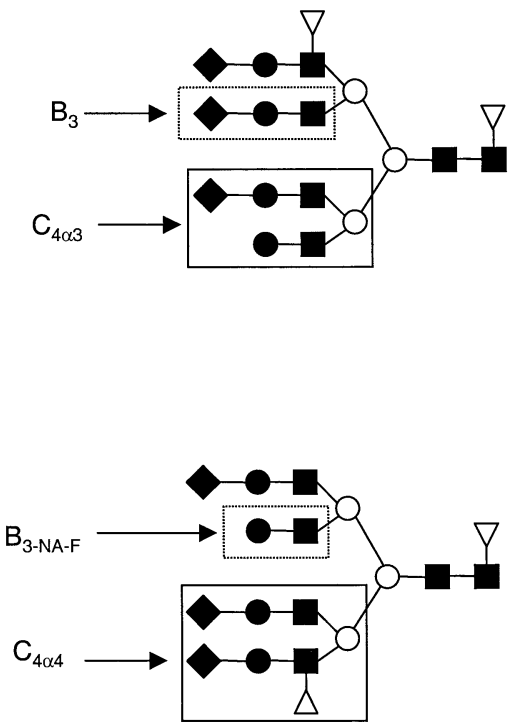

1612.6 uct of this enzymatic reaction was analyzed by the negative ion mode MS. It was shown to contain a mixture of mono-, di-, and trifucosylated N-glycans, represented by their triply and fourfold charged molecular ions (spectrum not shown). The new fucose moiety could potentially become attached to a shorter or to the longer chain antenna, and if on extended branch, either 


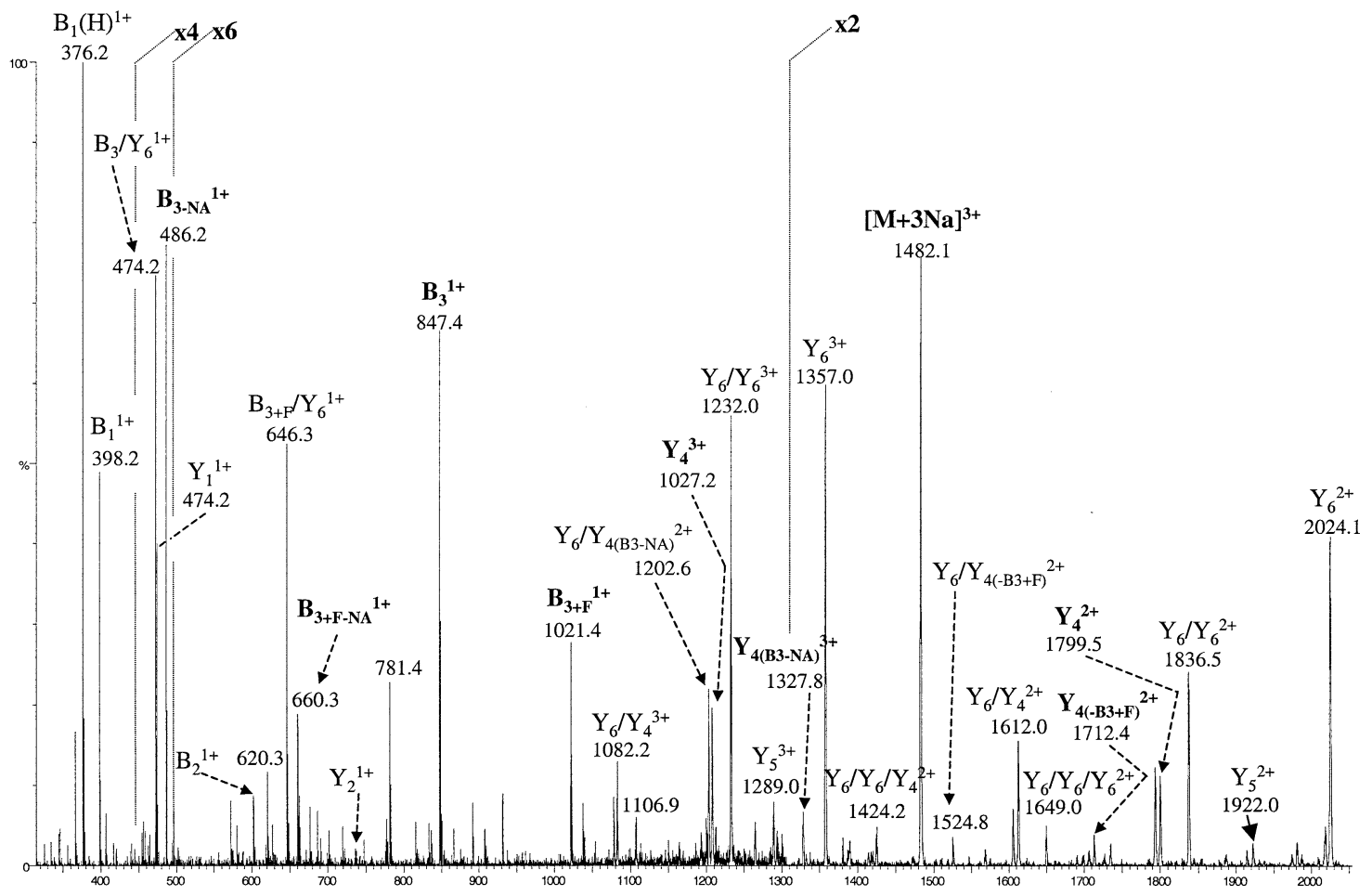

Figure 6. Positive ion MS/MS of the permethylated tetraantennary N-glycan containing three sialic acid, one core and one peripheral fucose moieties. The precursor ion was the molecular $[\mathrm{M}+3 \mathrm{Na}]^{3+}$ ion at $m / z 1482.1$.

CID (Figure 7). A vast number of fragment ions was generated, related to the size and complexity of this extended N-glycan structure. The low abundant singly charged ion at $m / z$ 1346.5, assigned to the internal cleavage ion $\mathrm{C}_{4 \alpha+\mathrm{F}} / \mathrm{Y}_{6}$, could be assigned to a fucosylated outer antenna without an additional LacNAc repeat unit after loss of a sialic acid moiety. The doubly charged ion at $m / z 745.3$ represents a $C_{4 \alpha}$ fragment ion, providing information for presence of the nonfucosylated antenna without additional LacNAc repeat units. $B_{6 \beta}$ or $C_{6 \beta}$ fragment ions were not observed in the spectrum. The antenna with an additional LacNAc repeat unit was found in its fucosylated as well as nonfucosylated glycoform as documented by internal fragment ion $B_{5 \beta^{\prime}+F} / Y_{6}$ as a singly charged signal at $m / z$ 875.3 and the $\mathrm{B}_{5 \beta^{\prime}}$ fragment ion at $m / z$ 1020.4. According to these ions FT VI lacks any preference for either short or elongated antenna of the N-glycan substrate for fucosylation: Both, these with or without additional LacNAc repeat unit, contained substoichiometric amounts of the fucose moiety. The presence of the $C_{4 \beta^{\prime}}$ fragment ion at $m / z 835.3$ and the absence of $C_{4 \beta^{\prime}+F}$ ion suggested that the $\alpha 1$-3fucosylation of the extended antenna is taking place only on the innermost GlcNAc moiety. Although some of diagnostic ions were of relatively low abundance, there were fully reproducible.

In order to explore the validity of these findings, the sample was permethylated and the resulting mixture of derivatives subjected to fragmentation analysis by tan- dem MS. In the positive ion mode MS/MS of the fourfold charged precursor ion at $\mathrm{m} / \mathrm{z} 1319.9$ (Figure 8) most of the fragment ions in the spectrum were of $B$ or $\mathrm{Y}$ type. The fragment ion $\mathrm{B}_{4 \alpha+\mathrm{F}} / \mathrm{Y}_{6}$ at $m / z 1661.9$ and the ion $B_{4 \alpha} / Y_{6}$ at $m / z 1486.8$ are diagnostic for the presence of fucosylated and nonfucosylated antennae without additional LacNAc repeat unit. Fucosylation of the antenna with an additional LacNAc repeat unit was documented by the $\mathrm{B}_{5 \beta^{\prime}+\mathrm{F}}$ ion at $m / z$ 1470.8. The fragment ion diagnostic for the attachment site of fucose in the extended antenna was $B_{4 \beta^{\prime}}$, along with its fucosylated analogue $B_{4 \beta^{\prime}+F}$, respectively. $B_{4 \beta^{\prime}}$ was observed in the spectrum as a low abundant singly charged ion at $\mathrm{m} / \mathrm{z}$ 1051.5, indicating the fucose attachment site at the inner GlcNAc rather than at the outer one. Although the diagnostic signals were in both native and permethylated N-glycan sample rather low abundant, they provided sufficient evidence for preferential fucosylation of the inner GlcNAc site on the antenna containing the extension by an additional LacNAc repeating unit by FT VI.

For sequencing of oligosaccharides, another approach not used in this study makes use of enzymatic degradation using specific exoglycosidases to cleave terminal monosaccharides from the nonreducing end, providing information on the identity, the linkage position, and the anomericity of the terminal monosaccharide residue. It has been shown to be useful in connection with MS mapping in cases where no tandem MS is available, since sequential degradation by either a sin- 


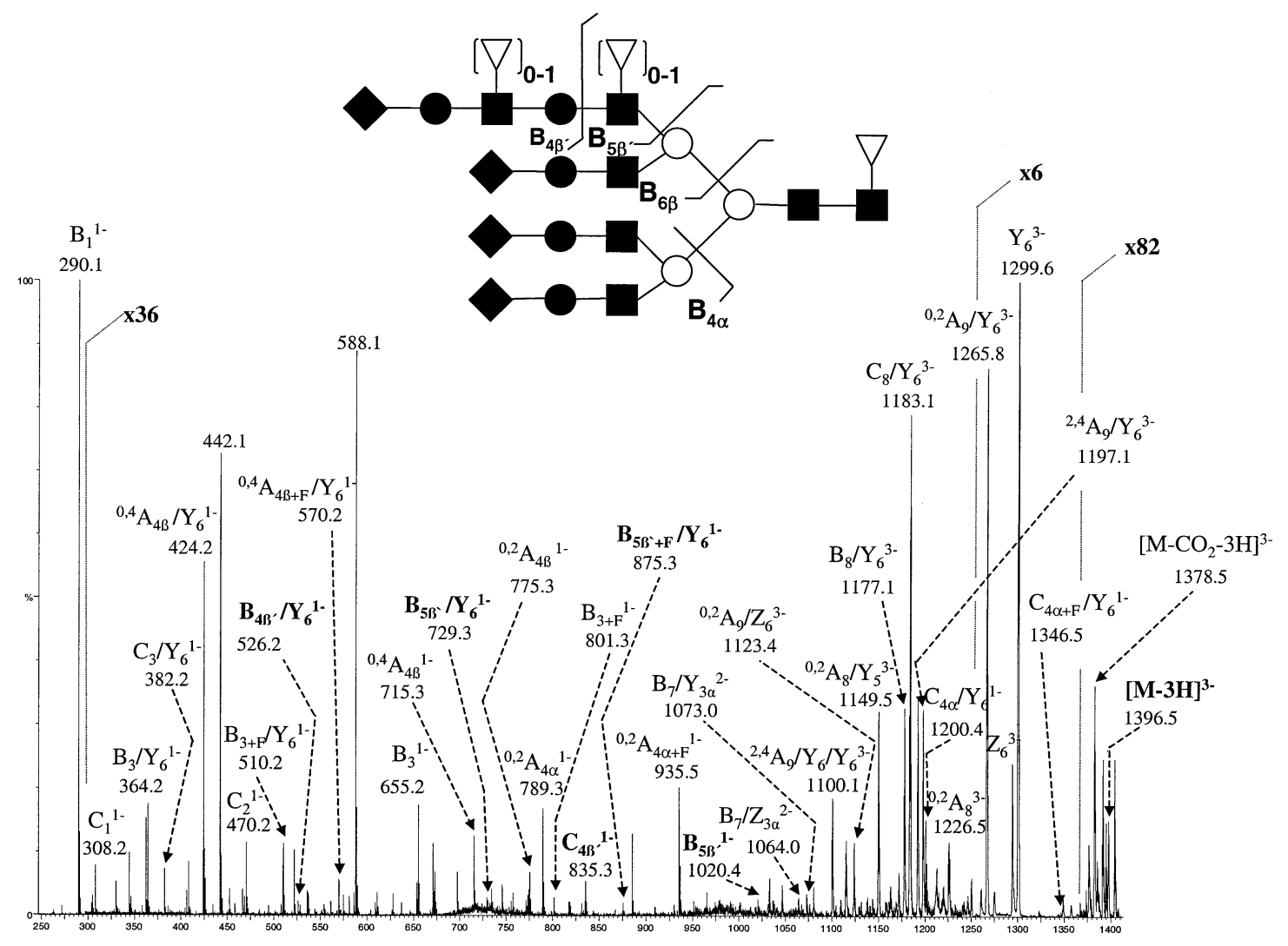

Figure 7. Negative ion MS/MS spectrum of the tetraantennary N-glycan containing one additional LacNAc repeat unit, four sialic acid, one core fucose and one peripheral fucose moieties. The triply charged ion at $\mathrm{m} / \mathrm{z} 1396.5$ was used as a precursor ion.

gle exoglycosidase or by defined enzyme mixtures (enzyme arrays) deliver the ladder sequence of an N-glycan [31] identifiable by MALDI MS [32, 33]. The most important prerequisites for enzymatic sequencing are high specificity and purity of the exoglycosidase, since contaminating glycosidase activities can give rise to misleading interpretations.

\section{Conclusions}

Using the ESI QTOF instrument, fragmentation analysis of single N-glycan components out of complex mixtures by low energy CID MS/MS has been investigated. The optimization of conditions used for MS/MS analysis was focused on putative branching, fucosylation and sialylation sites of $\mathrm{N}$-glycans in order to obtain structural information necessary for identification of specific epitopes. Tri- and tetraantennary N-glycans were fragmented, and fragment ions diagnostic for branching, fucose, and sialic acid position identified.

For analysis of native, underivatized, complex Nglycan mixtures, sialylated in most cases, the negative ion mode is advantageous over the positive mode for detection of molecular ions. The main reason is that molecular ions appear in most cases as singly or as multiply charged species without exchange of hydro- gen against sodium, or at only a low level of exchange. For fragmentation experiments by tandem MS, where the formation of cleavage ions in the negative ion mode CID is characterized as the one showing less neutral losses of water, the negative ion mode detection is preferable. B- and C-ions generally provide sufficient specific information about formation of biologically interesting epitopes on single branches, and are useful for determination of NeuAc and Fuc attachment sites. For the triantennary $\mathrm{N}$-glycan with the 6-linked antenna branched, the internal fragment ion $\mathrm{D}$ formed by loss of the 3-antenna, together with the chitobiose core $\left(\mathrm{B}_{5} / \mathrm{Y}_{3 \alpha}\right)$, was identified as the important diagnostic ion for the branching and antenna composition. For detection of core fucose, the cross-ring fragment ions ${ }^{0,2} \mathrm{~A}$ and ${ }^{2,4} \mathrm{~A}$, resulting from the reducing termini of $\mathrm{N}$-glycans, show the mass difference between fragment ions ${ }^{0,2} \mathrm{~A}$ and ${ }^{2,4} \mathrm{~A}$ of $206 \mathrm{Da}$ with the core-Fuc and $60 \mathrm{Da}$ without it.

To obtain realistic molecular maps from biological material, particular attention has to be paid to desorption conditions due to potential in-source degradation processes giving rise to in-source decay fragmentation of NeuAc or Fuc moieties. To explore the level of these processes control experiments are necessary. Derivatization of native $\mathrm{N}$-glycan mixtures by permethylation 


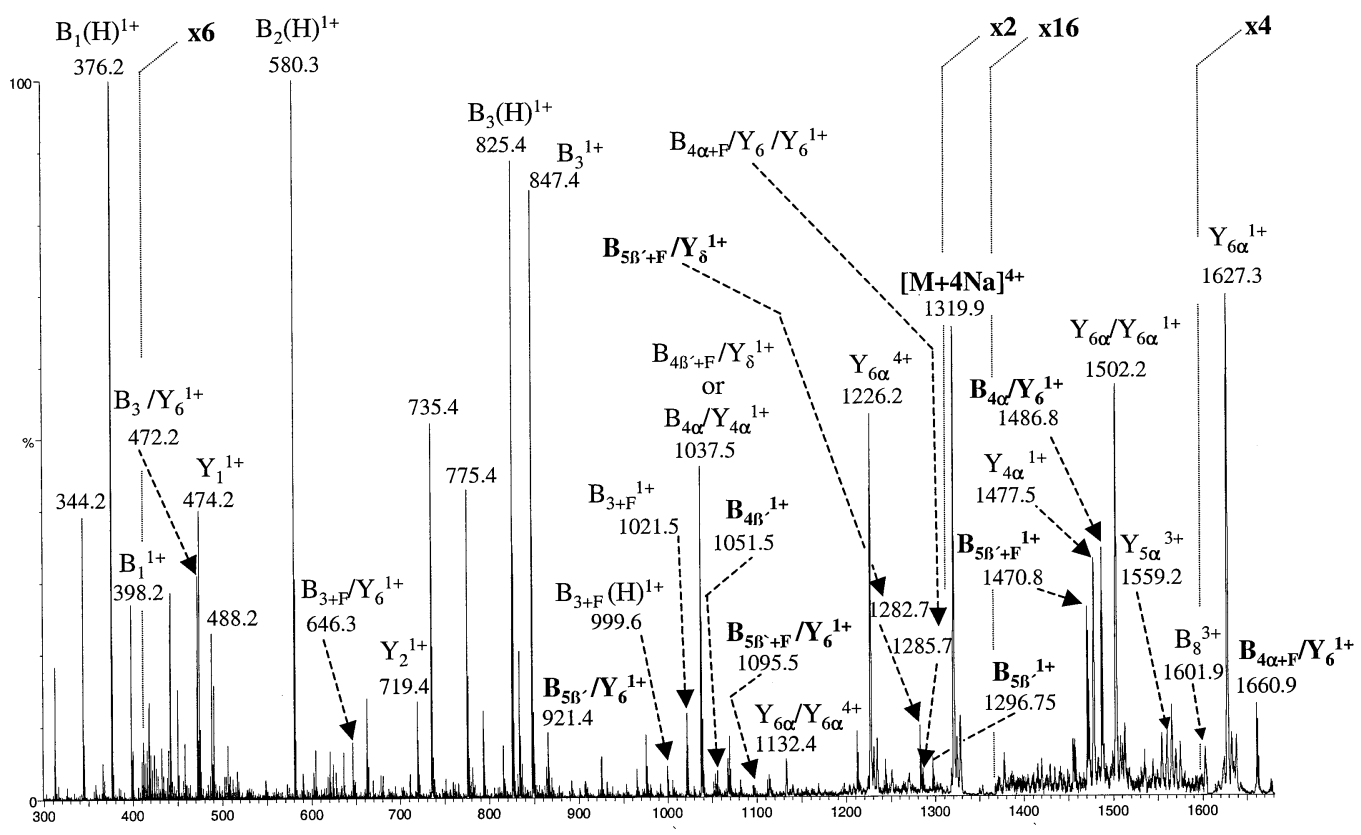

Figure 8. Positive ion MS/MS of the permethylated tetraantennary N-glycan containing one additional LacNAc repeat unit, four sialic acid, one core and one peripheral fucose moieties. The four-fold charged ion at $m / z 1319.9$ was used as a precursor ion.

provide some degree of stability, namely less glycosidic cleavages and neutral losses, though sufficient ionization degree. Derivatizations can be included in the experimental protocol if sufficient material for analysis, e.g., 10-15 pmoles, is available. In CID fragmentation experiments of permethylated substrates additional data for assignment of single and multiple cleavages are available due to distinct patterns created. In the present study, though, sialic acid moiety in the permethylated substrate was shown to be easily removable, giving rise to internal fragment ions, useful for identification of the NeuAc attachment site. On the other hand, undermethylation, which is a frequent feature in reaction of larger glycans, could provide misleading results in fragmentation analysis.

Mass spectrometry based strategy is unsurpassed as a tool for evaluation of acceptor specificity of single sialylated and nonsialylated antennae in multiantennary $\mathrm{N}$-glycans during fucosylation reaction in the Golgi. Well prepared native samples can be submitted directly to MS- and MS/MS-based identification of sialylated and fucosylated epitopes after in vitro glycosylation reactions for determination of their receptor specificity.

According to the here presented results in the reaction with FT VI the formation of $\mathrm{Le}^{\mathrm{x}}$ and $\mathrm{sLe}^{\mathrm{x}}$ motifs occurs with similar efficiency. If one of the antennae contains an additional LacNAc repeat it is obvious that the attachment of the fucose moiety by FT VI occurs preferentially on the inner GlcNAc on the antenna containing the extension by an additional LacNAc repeat unit.

\section{Acknowledgments}

DS highly appreciates the support by Professor Dr. D. Vestweber (University of Muenster, Germany). The ESI QTOF instrument was provided by a HbfG grant (Land Nordrhein-Westfalen, Germany) to JP-K.

Material presented in this publication is a part of the Ph.D. Thesis of DS, Faculty of Science, University of Münster, 2002.

\section{References}

1. Schachter, H. Glycoconj. J. 2000, 17, 465-483.

2. Nimtz, M.; Martin, W.; Wray, V.; Kloppel, K. D.; Augustin, J.; Conradt, H. S. Eur. J. Biochem. 1993, 213, 39-56.

3. Misaizu, T.; Matsuki, S.; Strickland, T. W.; Takeuchi, M.; Kobata, A.; Takasaki, S. Blood 1995, 86, 4097-4104

4. Schroter, S.; Derr, P.; Conradt, H. S.; Nimtz, M.; Hale, G.; Kirchhoff, C. J. Biol. Chem. 1999, 274, 29862-29873.

5. Treumann, A.; Lifely, M. R.; Schneider, P.; Ferguson, M. A. J. Biol. Chem. 1995, 270, 6088-6099.

6. Egge, H.; Peter-Katalinic, J. Mass Spectrom. Rev. 1987, 6, 331393.

7. Carr, S. A.; Reinhold, V. N.; Green, B. N.; Hass, J. Biomed. Mass Spectrom. 1985, 12, 288-295.

8. Gillece-Castro, B. L.; Burlingame, A. L. Methods Enzymol. 1990, 193, 689-712.

9. Reinhold, B. B.; Chan, S. Y.; Reuber, T. L.; Marra, A.; Walker, G. C.; Reinhold, V. N. J. Bacteriol. 1994, 176, 1997-2002.

10. Reinhold, V. N.; Reinhold, B. B.; Costello, C. E. Anal. Chem. Rev. 1995, 67, 1772-1784.

11. Charlwood, J.; Langridge, J.; Camilleri, P. Rapid Commun. Mass Spectrom. 1999, 13, 1522-1530.

12. Harvey, D. J. J. Am. Soc. Mass Spectrom. 2000, 11, 900-915.

13. Nimtz, M.; Grabenhorst, E.; Conradt, H. S.; Sanz, L.; Calvete, J. J. Eur. J. Biochem. 1999, 265, 703-718. 
14. Harvey, D. J.; Naven, T. J.; Kuster, B.; Bateman, R. H.; Green, M. R.; Critchley, G. Rapid Commun. Mass Spectrom. 1995, 9, 1556-1561.

15. Rouse, J. C.; Strang, A. M.; Yu, W.; Vath, J. E. Anal. Biochem. 1998, 256, 33-46.

16. Harvey, D. J.; Bateman, R. H.; Bordoli, R. S.; Tyldesley, R. Rapid Commun. Mass Spectrom. 2000, 14, 2135-2142.

17. Sheeley, D. M.; Reinhold, V. N. Anal. Chem. 1998, 70, 30533059.

18. Wheeler, S. F.; Harvey, D. J. Anal. Chem. 2000, 72, 5027-5039.

19. Schlenke, P.; Grabenhorst, E.; Nimtz, M.; Conradt, H. S. Cytotechnol. 1999, 30, 17-25.

20. Grabenhorst, E.; Nimtz, M.; Costa, J.; Conradt, H. S. J. Biol. Chem. 1998, 273, 30985-30994.

21. Nimtz, M.; Grabenhorst, E.; Gambert, U.; Costa, J.; Wray, V.; Morr, M.; Thiem, J.; Conradt, H. S. Glycoconj. J. 1999, 15, 873-885.

22. Packer, N. H.; Lawson, M. A.; Jardine, D. R.; Redmond, J. W. Glycoconj. J. 1998, 15, 737-747.

23. Kussmann, M.; Nordhoff, E.; Rehbek-Nielsen, H.; Haebel, S.; Rossel-Larsen, M.; Jakobsen, L.; Gobom, J.; Mirgorodskaya, E.;
Kroll-Kristensen, A.; Palm, L.; Roepstorff, P. J. Mass Spectrom. 1997, 32, 593-601.

24. Anumula, K. R.; Taylor, P. B. Anal. Biochem. 1992, 203, 101-108.

25. Kemminer, S. E.; Conradt, H. S.; Nimtz, M.; Sagi, D.; PeterKatalinic, J.; Diekmann, O.; Drmic, I.; Müthing, J. Biotechnol. Prog. 2001, 17, 809-821.

26. Harvey, D. J. Mass Spectrom. Rev. 1999, 18, 349-450.

27. Dell, A.; Thomas-Oates, J. In: Analysis of Carbohydrates by GLC and MC; Bierman, C. J.; McGinnis, G. D., Eds.; CRC Press: Boca Raton, 1989; pp 217-235.

28. Domon, B.; Müler, D. R.; Richter, W. J. Biomed. Environ. Mass Spectrom. 1990, 19, 390-392.

29. Garozzo, D.; Impallomeni, G.; Montaudo, G.; Spina, E. Rapid. Commun. Mass Spectrom. 1992, 6, 550-552.

30. Laine, R. A.; Yoon, E.; Mahier, T. J.; Abbas, S.; de Lappe, B.; Jain, R.; Matta, K. Biol. Mass Spectrom. 1991, 20, 505-514.

31. Kobata, A. Anal. Biochem. 1979, 100, 1-14.

32. Kuster, B.; Naven, T. J.; Harvey, D. J. J. Mass Spectrom. 1996, 31, 1131-1140.

33. Liedtke, S.; Geyer, R.; Geyer, H. Glycoconj. J. 1997, 14, 785-793. 